\title{
Angular distribution of electrons elastically scattered from $\mathrm{CH}_{4}$
}

\author{
T W Shyn and T E Cravens $\dagger$ \\ Space Physics Research Laboratory, The University of Michigan, Ann Arbor, MI 48109 , \\ USA
}

Received 30 May 1989, in final form 30 August 1989

\begin{abstract}
Differential elastic (vibrationally) scattering cross sections of $\mathrm{CH}_{4}$ by electron impact have been measured using a modulated crossed-beam method. The energy and angular range covered were from 5 to $50 \mathrm{eV}$ and from 12 to $156^{\circ}$, respectively. The integrated and momentum transfer cross sections were obtained from the differential cross sections. The present results are compared with the earlier data of Tanaka et al and with theoretical results of Lima et al and Jain and Thompson. Some discrepancies were found in the measurements and theoretical results.
\end{abstract}

\section{Introduction}

Methane is an important constituent of the atmospheres of the outer planets and comets. Electron interaction with $\mathrm{CH}_{4}$ or other gases plays a role in determining temperatures, electron densities and airglow or auroral emissions in these atmospheres. Understanding these atmospheric processes requires a knowledge of electron impact cross sections for $\mathrm{CH}_{4}$. Infrared emission $\left(\mathrm{CH}_{4}\right.$ bands) observed from Jupiter provides evidence that energetic auroral electrons are precipitating deep enough into atmosphere to reach the Jovian hydrocarbon layer ( $\mathrm{Kim} 1988$ ). Also, the upper atmosphere of Neptune might contain a few per cent of $\mathrm{CH}_{4}$ (Romani and Atreya 1988), in which case interaction of auroral electrons or photoelectrons with this gas will be important. Titan's upper atmosphere is known to be composed mainly of molecular nitrogen and methane (Hunten et al 1985), and electrons from the Saturnian magnetosphere can then collide with these gases, generating ionisation and airglow (Neubauer et al 1984). Some comets also contain significant amounts of methane, with which solar wind and atmospheric electrons can interact (cf Mendis et al 1985, Cravens et al 1987).

Electron impact cross section measurements for methane are required in order to understand the behaviour of both high-and low-energy electrons in many atmospheres. Vuskovic and Trajmar (1983) summarised the previous measurements on methane very well. In this paper, the discussion is restricted to the present energy range of total and elastic scattering cross sections. Total cross sections have been measured by Bruche (1927) from 0.85 to $49 \mathrm{eV}$ and by Barbarito et al (1979) from 0 to $16 \mathrm{eV}$. Three experimental determinations of absolute elastic cross sections have been made in the present energy region (5-50 eV). Rohr (1980) measured differential cross sections (DCs) in the energy range below $10 \mathrm{eV}$, over the angular range of $20-120^{\circ}$. Recently,

$\dagger$ Present address: Department of Physics and Astronomy, University of Kansas, Lawrence, KS 66045-2151, USA. 
Tanaka et al (1982) measured DCS in the energy range of 3 to $20 \mathrm{eV}$ for scattering angles from 30 to $140^{\circ}$. Vuskovic and Trajmar (1983) have measured relative DCS at 20,30 and $200 \mathrm{eV}$ and normalised their results to those of Tanaka et al at $100^{\circ}$ for 20 and $30 \mathrm{eV}$ impact.

There are more theoretical than experimental studies on electron impact collision with $\mathrm{CH}_{4}$. Gianturco and Thompson (1980) calculated total and momentum transfer cross sections below $1.5 \mathrm{eV}$ and DCS for $10 \mathrm{eV}$ by using a simple model in which electrons are scattered from a rigid molecule with exchange and polarisation effects. They compared their results of elastic scattering cross sections at $10 \mathrm{eV}$ with the relative DCs measured by Hughes and McMillen (1933) after being normalised at $90^{\circ}$, and found that the agreement is not so good. Jain and Thompson (1982) recently calculated the DCS by using a parameter-free polarisation potential for polyatomic molecules. The results of total cross sections are in relatively good agreement with the integrated cross sections of Tanaka et al. Also the DCS at $5 \mathrm{eV}$ is in good agreement with the measurements made by Tanaka et al, except for forward scatterings. Recently, Lima et al (1985) applied the Schwinger multichannel formulation with the static-plusexchange interaction to calculate integrated and differential cross sections in the energy range from 3 to $20 \mathrm{eV}$. Their calculated values of DCs are in good agreement with the measurements only at $7 \mathrm{eV}$, however, their integrated cross sections agree well with the data of Tanaka below $8 \mathrm{eV}$. More recently, Jain (1986) calculated total and DCS using a spherical model in the energy range of 0.1 to $500 \mathrm{eV}$. His results are in good agreement, in general, in forward scattering with the measurements but not in backward scattering.

As shown above, the existing measurements and theoretical calculations are not in good agreement and are quite fragmentary; thus, more extensive cross section information is still required. In this paper, we present the results of measurements of absolute differential elastic (vibrationally) cross sections for electron impact on methane for energies between $5 \mathrm{eV}$ and $50 \mathrm{eV}$, and scattering angles between 12 and $156^{\circ}$.

\section{Apparatus and procedure}

The schematic diagram of the apparatus used is shown in figure 1. A detailed description of the apparatus can be found elsewhere (Shyn et al 1972, 1988, Shyn 1980, Shyn and Sharp 1986). Briefly, the apparatus consists of an upper and a lower chamber. The two chambers are pumped differentially in order to maintain a low background pressure for the measurements in the lower chamber. The apparatus consists of three subsystems: (1) a methane source in the upper chamber, (2) a rotatable electron beam source, and (3) a fixed electron detector system on the vacuum chamber wall in the lower chamber.

The electron beam source, which is rotatable from $-90^{\circ}$ to $160^{\circ}$ continuously, consists of an electron gun, a $127^{\circ}$ electrostatic energy selector, two electron lens systems and two beam deflectors. The electron beam source can produce a current exceeding $10^{-8} \mathrm{~A}$ at energies above $10 \mathrm{eV}$ with $80 \mathrm{meV}$ full width at half maximum (FWHM). The half-angle spread of the elctron beam is less than $\pm 3^{\circ}$.

The detector system has two electron lens systems, two electrostatic energy analysers in series and a channeltron electron multiplier. The energy resolution of the detector system is beter than $80 \mathrm{meV}$ at FWHM.

The collimated $\mathrm{CH}_{4}$ beam from the upper chamber enters the lower chamber through a double skimmer located between the two chambers. The beam was modulated 


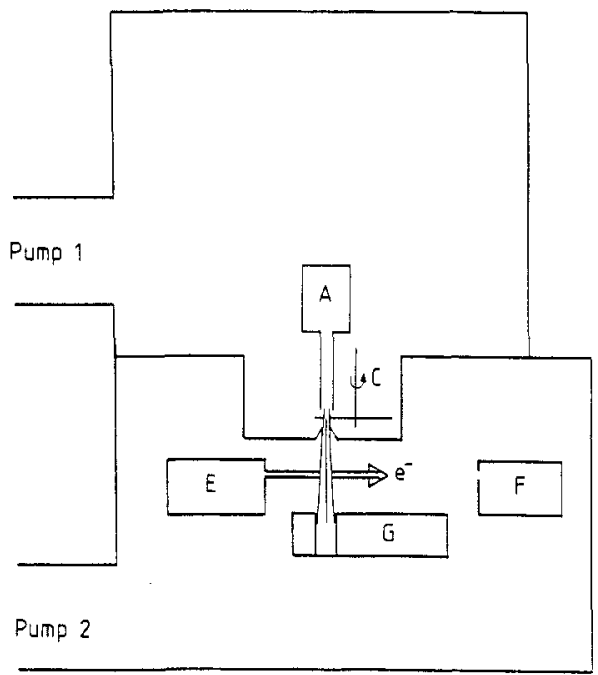

Figure 1. Schematic diagram of the apparatus. $\mathrm{A}, \mathrm{CH}_{4}$ beam source; $\mathrm{C}$, chopper; $\mathrm{E}$, electron beam source; $F$, electron detector; $G$, mass spectrometer.

at an audio frequency $(\approx 150 \mathrm{~Hz})$ by a toothed chopper wheel so that the pure beam signal can be separated from the background by using a phase-sensitive detector. Since the time constant of the present vacuum system for methane is estimated to be longer than $0.3 \mathrm{~s}$; therefore, it is believed there is negligible contribution to the beam signal from the background pressure, because the chopping period $(\simeq 7 \mathrm{~ms})$ is shorter than the time constant of the vacuum system by more than 40 times.

The incident electron beam of a given energy in the horizontal plane intersects with the vertically collimated and modulated neutral beam in an interaction region. The electrons elastically scattered from the modulated beam at a given angle are detected by the electron channeltron multiplier after energy analysis. The signals due to electrons scattered with the chopper open and closed are recorded by two counters, and the difference of these two signals gives the true scattered signal from the pure beam. This procedure is repeated for different angles and incident energies in order to obtain relative angular distributions. The impact energy scale is calibrated against the $19.3 \mathrm{eV}$ resonance in $\mathrm{He}$. It should be noted that, before the final cross section data of $\mathrm{CH}_{4}$ were taken, the angular distributions of electrons elastically scattered from helium for each incident energy were measured carefully and confirmed previous measurements. This was done in order to eliminate all systematic errors, including stray magnetic field effects and interaction volume effects. Three sets of Helmholtz coils reduce stray magnetic fields down to less than $20 \mathrm{mG}$ in all directions near the interaction region.

The relative angular distributions measured at each energy were put on absolute scale by normalisation with the elastic differential cross section of $\mathrm{He}$ reported by Shyn (1980) utilising a volume experiment (static gas background). The relative densities of the two gases ( $\mathrm{He}$ and $\mathrm{CH}_{4}$ ) were determined by measuring the corresponding pressures with an ion gauge, which was calibrated against an MKS Baratron pressure gauge.

The statistical uncertainty of each data point is $3 \%$. The uncertainty in the normalisation procedure is estimated to be $10 \%$. Therefore, the overall uncertainty 
of the present results is about $14 \%$ including the uncertainty of the He cross sections $(10 \%)$.

\section{Experimental results and discussion}

Absolute differential elastic cross sections have been measured at the following incident energies: $5.0,10,15,20,30$ and $50 \mathrm{eV}$. The results are summarised in table 1 , including the integrated and momentum transfer cross sections.

Figure 2 shows DCs at $5.0 \mathrm{eV}$ impact energy along with the results of Tanaka et al and Rohr. The theoretical results of Jain are also included. The DCs indicates a dominant $\mathrm{D}$-wave character. The previous measurements are in good agreement with the present results except that their minima are shifted toward smaller angles $\left(45^{\circ}\right.$ and

Table 1. Differential elastic cross sections, integrated and momentum transfer cross sections of methane. The numbers in parentheses are extrapolated data points. $\sigma_{\mathrm{i}}$ and $\sigma_{\mathrm{m}}$ are given in units of $10^{-16} \mathrm{~cm}^{2}$.

\begin{tabular}{|c|c|c|c|c|c|c|c|c|c|c|c|c|c|c|c|c|}
\hline \multicolumn{17}{|c|}{$\mathrm{d} \sigma / \mathrm{d} \Omega 10^{-17} \mathrm{~cm}^{2} \mathrm{sr}^{-1}$} \\
\hline $\begin{array}{c}\phi(\mathrm{deg}) \\
E(\mathrm{eV})\end{array}$ & & 24 & 36 & 48 & 60 & 72 & 74 & 96 & 108 & 120 & 132 & 144 & 156 & 168 & $\sigma_{\mathrm{i}}$ & $\sigma_{\mathrm{mt}}$ \\
\hline 5.0 & 44.2 & 27.5 & 16.1 & 13.3 & 12.3 & 13.5 & 15.0 & 13.3 & 9.2 & 3.6 & 2.3 & 4.2 & 6.7 & $(10.5)$ & 14.8 & 10.7 \\
\hline 10 & 115.2 & 66.7 & 38.1 & 25.4 & 14.6 & 9.4 & 7.3 & 5.2 & 2.9 & 2.1 & 3.5 & 8.0 & 14.0 & $(29.5)$ & 19.8 & 10.5 \\
\hline 15 & 154.8 & 74.3 & 30.4 & 19.9 & 10.3 & 6.0 & 3.4 & 2.9 & 1.9 & 2.3 & 4.5 & 6.9 & 9.2 & $(12.4)$ & 17.6 & 7.5 \\
\hline 20 & 169.1 & 73.3 & 24.6 & 13.2 & 6.7 & 4.2 & 2.8 & 1.8 & 1.6 & 2.2 & 3.3 & 4.9 & 5.7 & $(7.2)$ & 15.3 & 5.4 \\
\hline 30 & 165.0 & 52.3 & 13.4 & 6.7 & 3.8 & 2.5 & 1.7 & 1.1 & 1.2 & 1.7 & 2.5 & 3.2 & 3.7 & $(4.8)$ & 11.3 & 3.5 \\
\hline 50 & 82.3 & 18.0 & 4.4 & 3.0 & 1.6 & 0.85 & 0.49 & 0.48 & 0.62 & 1.2 & 1.8 & 1.9 & 2.1 & (2.3) & 5.0 & 1.8 \\
\hline
\end{tabular}

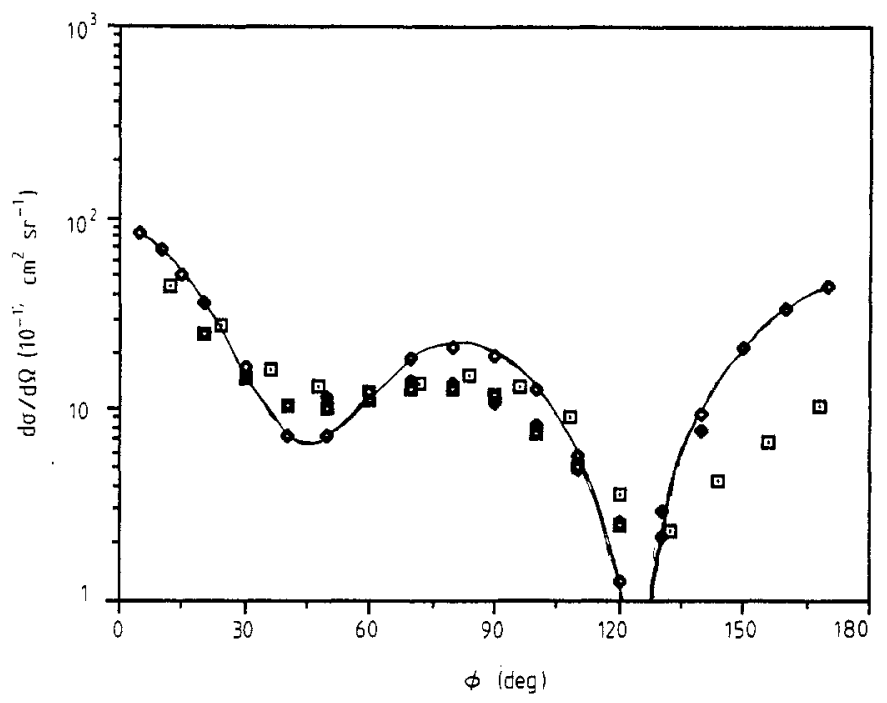

Figure 2. Measured angular distribution of elastic cross sections at $5.0 \mathrm{eV}$ ([). The measurements of Tanaka et al (1982) ( ) and Rohr (1980) ( $\mathbf{0})$ and the theoretical results of Jain (1986) (-૪-) are also shown. 
$120^{\circ}$ ) compared with the present results at 60 and $135^{\circ}$. The theoretical results of Jain agree with the present results in general shape but his results have larger values near $90^{\circ}$ and backward scattering than the present results by more than a factor of two except near $120^{\circ}$. The present results show a shallower distribution than those of Jain around $110-120^{\circ}$. This may be due to the contributions from the rotational excitations $(\Delta J=0)$ as Jain mentioned. The present DCS at 10 and $15 \mathrm{eV}$ exhibit strong forward and backward scattering with a small inflection near $90^{\circ}$ and with the minimum at $120^{\circ}$ and $105^{\circ}$, respectively.

Figure 3 is the same as figure 2 but for $20 \mathrm{eV}$ impact. The results of Tanaka et al agree with the present results except near $90^{\circ}$. Both results show a typical P-wave shape. Jain's results have the same tendencies as at $5.0 \mathrm{eV}$.

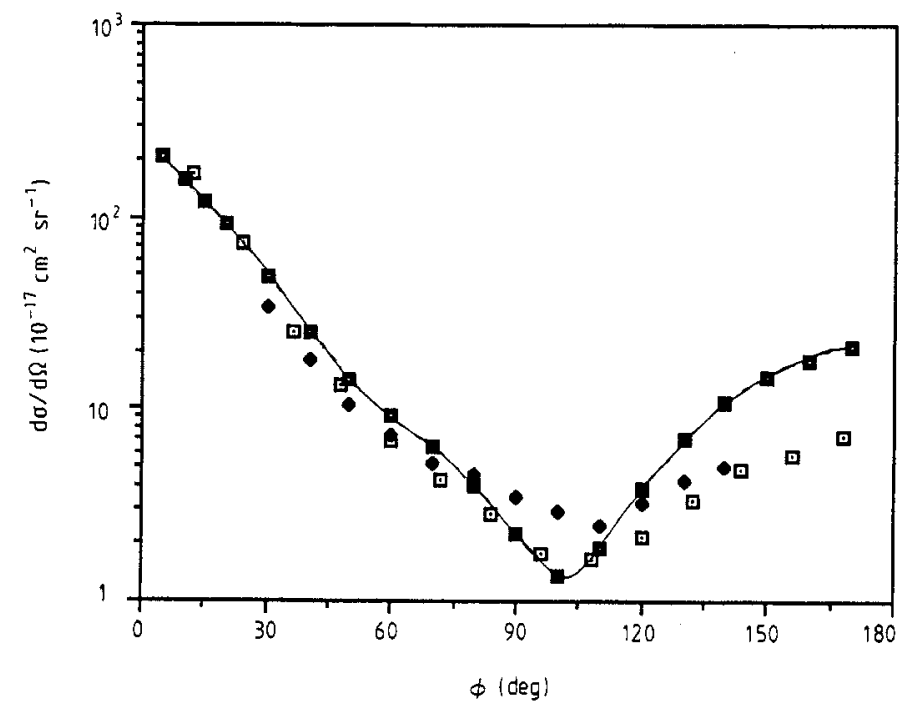

Figure 3. Measured angular distribution of elastic cross section at $20 \mathrm{eV}(\square)$. The measurements of Tanaka et al (1982) ( ) and the theoretical results of Jain (1986) (-_—) are also shown.

Figure 4 shows DCS of $50 \mathrm{eV}$ along with the results of Jain. Very strong forward scattering and a mild backward peak with a minimum near $90^{\circ}$ are evident. The theoretical results of Jain agree very well in shape with the present results but their absolute values are larger than the present results by approximately a factor of two. Generally, the minimum in the DCs moves to smaller scattering angles as the incident energy increases.

Figure 5 shows integrated cross sections along with those of Tanaka et al and the theoretical values of Lima et al. The total cross sections of Bruche (1927) and Barbarito et al (1979) are also shown. The integrated cross sections were obtained by integrating DCS over scattering angles after exponentially extrapolating to $180^{\circ}$. Since there is a $\sin \phi$ factor in the integration over solid angles, the expected uncertainty due to the extrapolations to extreme angles is less than a few per cent. Agreement is relatively good between the two sets of measured integrated cross sections. Both measurements show a broad maximum between 5 and $10 \mathrm{eV}$. It is noted that Tanaka et al used the relative flow technique for the normalisation of relative cross sections and an effective range theory expansion in a phaseshift analysis was used to extrapolate the data to 


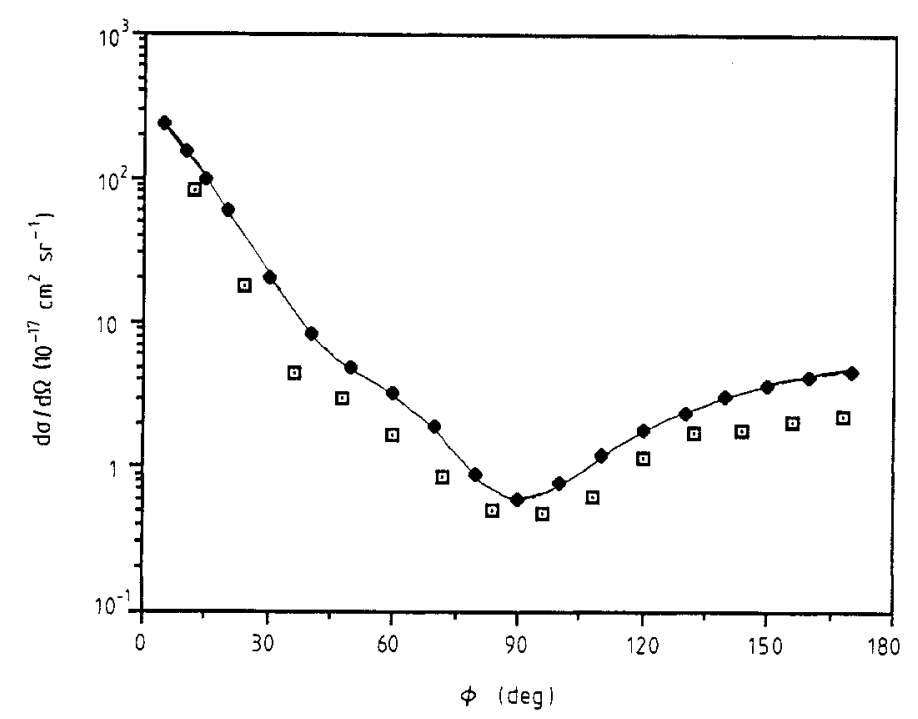

Figure 4. Measured angular distribution of elastic cross section at $50 \mathrm{eV}$ impact energy (). The theoretical results of Jain (1986) (- - ) are also shown.

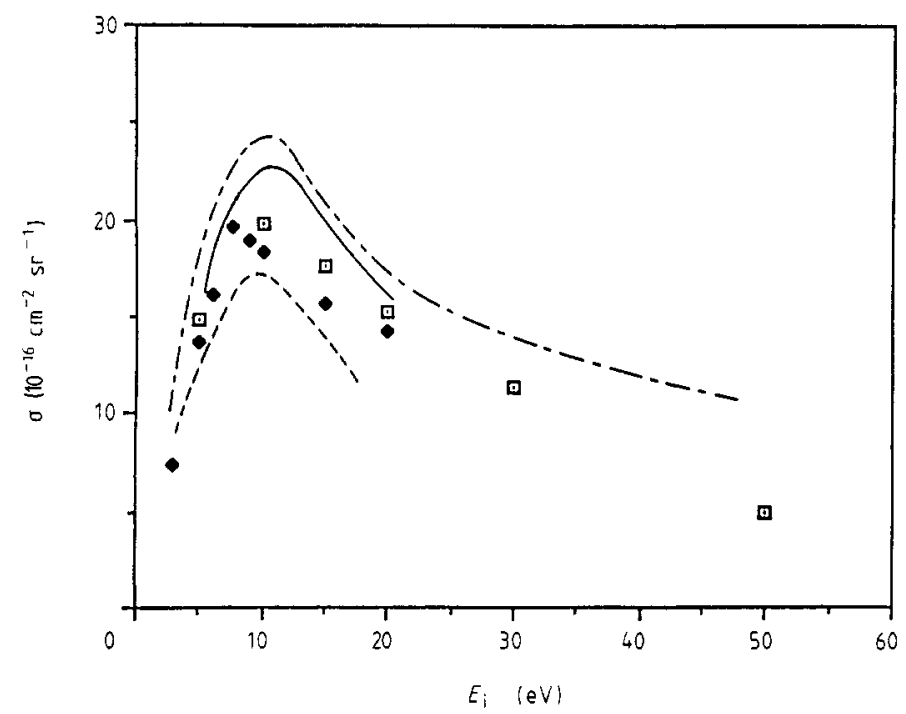

Figure 5. Total and integrated cross sections of methane from this paper $(\square)$ and the results of Tanaka et al (1982) ( ). Also shown are the total cross sections measured by Bruche (1927) (- - - ) and Barbarito et al (1979) (---) and the theoretical results of Lima et al (1985) (-) (integrated).

the extreme angles. The difference between the total cross sections of Bruche and the present integrated cross sections is the sum of inelastic cross sections. However, the results of Barbarito et al are smaller than the present integrated cross sections for all incident energies and, therefore, something must be wrong with their measurements. The theoretical results of Lima $e t a l$ are in agreement with the present results at 5 and $20 \mathrm{eV}$ impact energy but they have larger values between these two energies. The 
theoretical values of Jain indicate the same trend as Lima et al and his values are almost the same as the values of Bruche's total cross sections.

Finally, momentum transfer cross sections have been calculated from the present DCs and are shown in figure 6 along with the results of Tanaka et al. The results of Tanaka et al are larger than the present results by about $20 \%$ except at $15 \mathrm{eV}$, even though their DCS are smaller than present results by about $10 \%$. Perhaps something is wrong with their extrapolations of the DCS to $0^{\circ}$ and $180^{\circ}$.

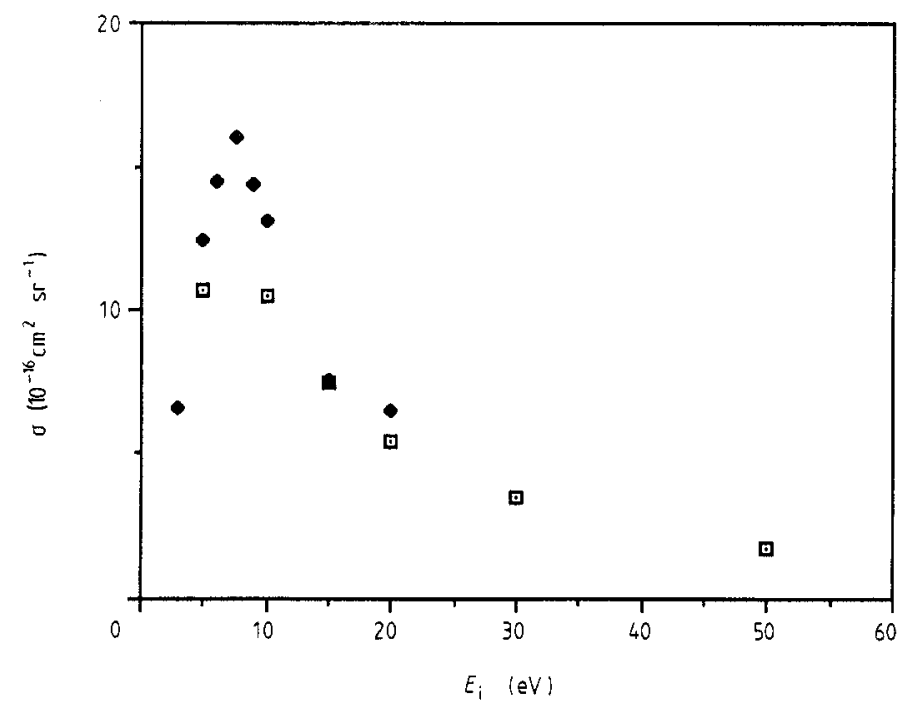

Figure 6. Momentum transfer cross sections from this paper ( $\square$ ) and the results of Tanaka et al (1982) (৬).

Measured electron impact cross sections, such as those presented in this paper, are needed in order to calculate electron fluxes in planetary atmospheres, such as that of Titan, which contain significant amounts of methane (Hunten et al 1985). For example, the two-stream method of calculating atmospheric superthermal electron fluxes (as a function of energy) requires elastic backscattering probabilities (cf Banks and Kockarts 1973). Backscattering probabilities for elastic scattering for $\mathrm{CH}_{4}$ can easily be calculated from the measured differential cross sections presented in this paper.

\section{Acknowledgment}

This work was supported by NASA Grant No NAGW-938.

\section{References}

Banks P M and Kockards G 1973 Aeronomy (New York: Academic)

Barbarito E, Basta M and Calicchio M 1979 J. Chem. Phys. 7154

Bruche E 1927 Ann. Phys., Lpz. 831065

Cravens T E, Kozyra J U, Nagy A F, Combosi T I and Kurtz M 1987 J. Geophys. Res. 927341

Gianturco F A and Thompson D G 1980 J. Phys. B: At. Mol. Phys. 13613 
Hughes and McMillen 1933 Phys. Rev. 44876

Hunten D M, Tomasko M G, Flasar F M, Samuelson R E, Strobel D F and Stevens D J 1984 Saturn ed T Gehrels and M S Matthews (Tucson: University of Arizona Press) pp 671-760

Jain A 1986 Phys. Rev. A $\mathbf{3 4} 3707$

Jain A and Thompson D G 1982 J. Phys. B: At. Mol. Phys. 15 L631

Kim S J 1988 Icarus 75399

Lima M A, Gibson T L, Huo W M and McKoy V 1985 Phys. Rev. A 322696

Mendis D A, Houpis H L F and Marconi M L 1985 Fund. Cosmic Phys. 101

Neubauer F M, Gurnett D A, Schudder J D and Hartle R E 1984 Saturn ed T Gehrels and M S Matthews (Tucson: University of Arizona Press) pp 671-760

Rohr K 1980 J. Phys. B: At. Mol. Phys. 134897

Romani P N and Atreya S K 1988 Icarus 74424

Shyn T W, Stolarski R S and Carignan G R 1972 Phys. Rev. A 61002

Shyn T W 1980 Phys. Rev. A 22916

Shyn T W, Cho S Y and Cravens T E 1988 Phys. Rev. A 38678

Shyn T W and Sharp W E 1986 J. of Geophys. Res. 911691

Tanaka H, Okada T, Boesten L, Suzuki T, Yamamoto T and Kubo M 1982 J. Phys. B: At. Mol. Phys. 153305

Vuskovic L and Trajmar S 1983 J. Chem. Phys. 784947 